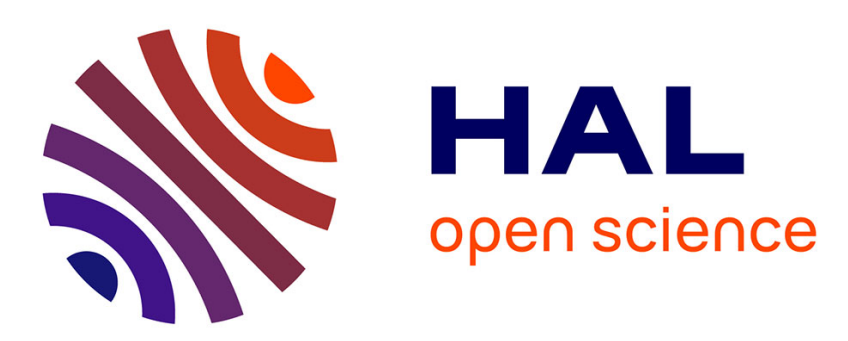

\title{
Semi-Lagrangian schemes for mean field game models
}

Elisabetta Carlini, Francisco José Silva

\section{To cite this version:}

Elisabetta Carlini, Francisco José Silva. Semi-Lagrangian schemes for mean field game models. 2014. hal-00913737

\section{HAL Id: hal-00913737 \\ https: / hal-unilim.archives-ouvertes.fr/hal-00913737}

Preprint submitted on 22 Jan 2014

HAL is a multi-disciplinary open access archive for the deposit and dissemination of scientific research documents, whether they are published or not. The documents may come from teaching and research institutions in France or abroad, or from public or private research centers.
L'archive ouverte pluridisciplinaire HAL, est destinée au dépôt et à la diffusion de documents scientifiques de niveau recherche, publiés ou non, émanant des établissements d'enseignement et de recherche français ou étrangers, des laboratoires publics ou privés. 


\section{Semi-Lagrangian schemes for mean field game models}

\author{
E. Carlini
}

\begin{abstract}
In this work we consider first and second order Mean Field Games (MFGs) systems, introduced in [18], [19], [20]. For the first order case, we recall a fully-discrete SemiLagrangian (SL) scheme introduced in [9] and its main properties. We propose the natural extension of this scheme for the second order case and we present some numerical simulations.

\section{INTRODUCTION}

Let us consider the system

$$
\begin{aligned}
-\partial_{t} v-\sigma \Delta v+H(D v) & =F(x, m(t)), \quad \text { in } Q, \\
\partial_{t} m-\sigma \Delta m-\operatorname{div}(D H(D v) m) & =0, \quad \text { in } Q, \\
v(x, T)=G(x, m(T)) \quad \text { for } x \in \mathbb{R}^{d} & , m(0)=m_{0},
\end{aligned}
$$
\end{abstract}

where $D$ denotes the space gradient, $Q=\mathbb{R}^{d} \times(0, T), T>0$, $\sigma \in \mathbb{R}^{+}$and $m_{0}$ is a probability measure on $\mathbb{R}^{d}$. System (I.1), as well as its stationary version, were introduced by Lasry and Lions in [18], [19], [20] in order to model Nash equilibria for differential games with a large numbers of small players (see also the survey [15] about the various applications of this theory in economics, natural resources management, collective behavior, etc). Formally, the first equation, together with its final condition, describes the value function of an average or generic player, whereas the second equation models the evolution of the initial distribution $m_{0}$ of the players if they follow the optimal strategy of the generic player.

Under suitable assumptions over the data (see section II) (I.1) admits at least one solution. More precisely, if $\sigma \neq 0$ system (I.1) has a classical solution $(v, m)$, whereas if $\sigma=0$ there exists $(v, m)$ such that the first equation is satisfied in the viscosity sense and the second equation is satisfied in the distributional sense. Uniqueness of such solutions can be established under an additional monotonicity assumption over $F$ and the final datum $G$ (see (II.1)).

Finite-difference schemes to solve (I.1) when $\sigma \neq 0$, have been proposed by [2], [3] and the first convergence results have been obtained recently in [13], [14], for the case when $H$ is quadratic, and in [1], for the general case. Let us mention that these methods allow to treat the case of local interactions i.e. when the function $F$ is of the form $F(x, m)=f(m(x))$ for every absolutely continuous probability measure $m$. In the same context of local interactions, let us also mention the article [16], where a numerical method, based on the optimal control of parabolic equations,

\footnotetext{
Dipartimento di Matematica, Sapienza Università di Roma (carlini@mat.uniromal.it) +3906 49913214.

XLIM - DMI UMR CNRS 7252 Faculté des Sciences et Techniques, Université de Limoges (francisco.silva@unilim.fr) +33 5 87506787
}

\author{
F. J. Silva
}

is analyzed in detail. For the case $\sigma=0$ the first step in the approximation analysis was achieved in [7], where a semidiscrete in time approximation was proved to converge to the solution of the problem. Recently, in [9] an implementable SL scheme was proposed and studied in detail.

Our aim in this article is to present some recent advances in the numerical discretization of (I.1) for both: first order and second order systems. We concentrate ourself on fully discrete SL discretizations, as an interesting alternative to finite-difference schemes, and their main properties. More precisely, we will summarize the scheme proposed in [9], which is the first paper that deals with the numerical analysis of the first order system, and the main results obtained. Next, we propose a natural extension to the second order case and we display some simulations which show that our scheme behaves well in practice. Theoretical studies such as convergence results for our scheme in the second order case, are the authors current subject of research.

For the sake of clarity, in this article we focus our attention on numerical methods for the quadratic case, i.e. when

$$
H(p)=\frac{1}{2}|p|^{2} .
$$

Nevertheless, our schemes, as well as their main properties, can be extended to more general problems (see e.g. section $\mathrm{VI})$.

The article is organized as follows: We begin in section II by recalling the standard assumptions for the data of (I.1). Next, we review in section III the SL scheme introduced in [9] for the first order case and the main results: existence of a solution of the scheme and convergence to the solution of (I.1). In section IV we propose a SL scheme for the second order case and we provide in section $\mathrm{V}$ some numerical tests for both types of systems. Finally, we discuss in section VI some natural generalizations of our scheme for more complicated problems.

\section{ASSUMPTIONS AND PRELIMINARIES}

We denote by $\mathcal{P}_{1}$ the space of probability measures over $\mathbb{R}^{d}$ endowed with the Kantorvich distance:

$d_{1}(\mu, \nu)=\sup \left\{\int_{\mathbb{R}^{d}} \phi(x) \mathrm{d}[\mu-\nu](x) ; \phi\right.$ is 1-Lipschitz $\}$.

In what follows we will suppose that $H$ is given by (I.2) and that

- $F$ and $G$ are continuous over $\mathbb{R}^{d} \times \mathcal{P}_{1}$.

- We have the following regularity for $F$ and $G$

$$
\|F(\cdot, m)\|_{C^{2}}+\|G(\cdot, m)\|_{C^{2}} \leq C .
$$


- We have that $m_{0}$ is absolutely continuous, with density still denoted by $m_{0} \in L^{\infty}\left(\mathbb{R}^{d}\right)$, and has compact support.

Note that the second assumption implies that $F$ and $G$ can be interpreted as smoothing operators in the sense that they transform probability measures into $C^{2}$ functions.

We have (see [19], [20], [21], [8])

Theorem 2.1: If $\sigma \neq 0$ system (I.1) has a classical solution $(v, m)$. If $\sigma=0$ then there exists $(v, m) \in$ $W^{1, \infty}(Q) \times C\left([0, T] ; \mathcal{P}_{1}\right)$ such that the first equation in (I.1) is satisfied in the viscosity sense, where the second equation is satisfied in the distributional sense.

A uniqueness result can be obtained assuming the following monotonicity condition: for all $m_{1}, m_{2} \in \mathcal{P}_{1}$ we have

$$
\begin{aligned}
& \int_{\mathbb{R}^{d}}\left[F\left(x, m_{1}\right)-F\left(x, m_{2}\right)\right] \mathrm{d}\left[m_{1}-m_{2}\right](x) \geq 0 \\
& \int_{\mathbb{R}^{d}}\left[G\left(x, m_{1}\right)-G\left(x, m_{2}\right)\right] \mathrm{d}\left[m_{1}-m_{2}\right](x) \geq 0 .
\end{aligned}
$$

We have (see [19], [20], [21], [8])

Theorem 2.2: Under the additional condition (II.1) the solution of (I.1) in Theorem 2.1 is unique.

Remark 2.1: The above results remain valid if $F$ depends also explicitly on the time variable $t$. However, in order to simplify the notations we will not consider this dependence in the presentation of our results.

In the rest of the article we focus our attention on discretizations of (I.1). For the sake of simplicity, we will present our schemes in the case when the space dimension is one. Nevertheless, the schemes can be written in the case of a general space dimension $d$ (see [9]).

Given $h, \rho>0$, let us define a space lattice $\mathcal{G}_{\rho}$ and a time-space grid $\mathcal{G}_{\rho, h}$ as

$$
\mathcal{G}_{\rho}:=\left\{x_{i}=i \rho, i \in \mathbb{Z}\right\} \quad \mathcal{G}_{\rho, h}:=\mathcal{G}_{\rho} \times\left\{t_{k}\right\}_{k=0}^{N},
$$

where $t_{k}=k h(k=0, \ldots, N)$ and $t_{N}=N h=T$. Let us call $B\left(\mathcal{G}_{\rho}\right)$ and $B\left(\mathcal{G}_{\rho, h}\right)$ the space of bounded functions defined on $\mathcal{G}_{\rho}$ and $\mathcal{G}_{\rho, h}$, respectively. Given $f \in B\left(\mathcal{G}_{\rho}\right)$ and $g \in B\left(\mathcal{G}_{\rho, h}\right)$ we define $f_{i}:=f\left(x_{i}\right), g_{i, k}:=g\left(x_{i}, t_{k}\right)$. Let us consider the following linear interpolation operator

$$
I[f](\cdot):=\sum_{i \in \mathbb{Z}} f_{i} \beta_{i}(\cdot) \text { for } f \in B\left(\mathcal{G}_{\rho}\right),
$$

where $\left\{\beta_{i} ; i \in \mathbb{Z}\right\}$ are defined by

$$
\beta_{i}(x):=\max \left\{\left[1-\left|x-x_{i}\right| / \rho, 0\right\} .\right.
$$

We have that $\beta_{i}(x)$ is a continuous function with support $\left[x_{i}-\rho, x_{i}+\rho\right]$ and satisfies $0 \leq \beta_{i} \leq 1, \beta_{i}\left(x_{j}\right)=\delta_{i j}$ (the Kronecker symbol) and $\sum_{i \in \mathbb{Z}} \beta_{i}(x)=1$.

\section{A FULLY DISCRETE SL SCHEME FOR THE FIRST ORDER MFG SYSTEM}

In this section we recall the scheme proposed in [9] for the system

$$
\begin{aligned}
& -\partial_{t} v+\frac{1}{2}|D v|^{2}=F(x, m(t)), \text { in } Q, \\
& \partial_{t} m-\operatorname{div}(D v m)=0, \quad \text { in } Q, \\
& v(x, T)=G(x, m(T)) \quad \text { for } x \in \mathbb{R}, \quad m(0)=m_{0} .
\end{aligned}
$$

Given $\mu \in C\left([0, T] ; \mathcal{P}_{1}\right)$, we denote by $v[\mu]$ the unique viscosity solution (see [5]) of the following Hamilton-JacobiBellman (HJB) equation

$$
\begin{aligned}
& -\partial_{t} v+\frac{1}{2}|D v|^{2}=F(x, \mu(t)), \quad \text { in } Q, \\
& v(x, T)=G(x, \mu(T)) \quad \text { for } x \in \mathbb{R}
\end{aligned}
$$

and by $m[\mu]$ the unique solution in the distributional sense, (see [4]), of the continuity equation

$$
\begin{aligned}
& \partial_{t} m-\operatorname{div}(D v m)=0, \text { in } Q, \\
& m(0)=m_{0} \in \mathcal{P}_{1} .
\end{aligned}
$$

To find a pair $(v, m)$ such that (III.1) holds true is thus equivalent to

Find $\mu \in C\left([0, T] ; \mathcal{P}_{1}\right)$ such that $m[\mu]=\mu$.

We present first the discretization of both equations in (III.1) separately and then the corresponding discretization of (III.4).

\section{A. The fully-discrete scheme for the HJB equation}

For a given $\mu \in C\left([0, T] ; \mathcal{P}_{1}\right)$ let us define $v \in B\left(\mathcal{G}_{\rho, h}\right)$ using the following SL scheme for (III.2):

$$
\left\{\begin{array}{l}
v_{i, k}=S_{\rho, h}[\mu]\left(v_{\cdot, k+1}, i, k\right) \quad \forall k=0, \ldots, N-1, \\
v_{i, N}=G\left(x_{i}, \mu\left(t_{N}\right)\right),
\end{array}\right.
$$

with $S_{\rho, h}[\mu]: B\left(\mathcal{G}_{\rho}\right) \times \mathbb{Z} \times\{0, \ldots, N-1\} \rightarrow \mathbb{R}$ given by

$$
\begin{aligned}
S_{\rho, h}[\mu](f, i, k):= & \inf _{\alpha \in \mathbb{R}}\left[\frac{1}{2} I[f]\left(x_{i}-h \alpha\right)+\frac{1}{2} h|\alpha|^{2}\right] \\
& +h F\left(x_{i}, \mu\left(t_{k}\right)\right) .
\end{aligned}
$$

This type of SL scheme for HJB equations has been studied extensively in the recent years (see e.g. [11] and the references therein for a detailed exposition). Let us set

$$
v_{\rho, h}[\mu](x, t):=I\left[v_{\cdot,\left[\frac{t}{h}\right]}\right](x) \quad \text { for all }(x, t) \in Q,
$$

which can be seen as an extension to $Q$ of $v$ defined by (III.5) in the time-space grid $\mathcal{G}_{\rho, h}$. We have the following convergence result (see [9, Theorem 4.5]):

Theorem 3.1: Let $\left(\rho_{n}, h_{n}\right) \rightarrow 0$ be such that $\frac{\rho_{n}^{2}}{h_{n}} \rightarrow 0$. Then, for every sequence $\mu_{n} \in C\left([0, T] ; \mathcal{P}_{1}\right)$ such that $\mu_{n} \rightarrow \mu$ in $C\left([0, T] ; \mathcal{P}_{1}\right)$, we have that $v_{\rho_{n}, h_{n}}\left[\mu_{n}\right] \rightarrow v[\mu]$ uniformly over compact sets of $Q$.

\section{B. The fully-discrete scheme for the continuity equation}

Given $\rho \in C_{c}^{\infty}(\mathbb{R})$ such that $\rho \geq 0$ and $\int_{\mathbb{R}} \rho(x) \mathrm{d} x=1$, for $\varepsilon>0$ consider the mollifier $\rho_{\varepsilon}(x):=\frac{1}{\varepsilon} \rho\left(\frac{x}{\varepsilon}\right)$ and set

$$
v_{\rho, h}^{\varepsilon}[\mu](\cdot, t):=\rho_{\varepsilon} * v_{\rho, h}[\mu](\cdot, t) .
$$

Let us consider the discrete flow

$$
\Phi_{i, k, k+1}^{\varepsilon}[\mu]:=x_{i}-h D v_{\rho, h}^{\varepsilon}[\mu]\left(x_{i}, t_{k}\right) .
$$

We define the following scheme in the time-space grid $\mathcal{G}_{\rho, h}$

$$
\begin{aligned}
m_{i, k+1}^{\varepsilon}[\mu] & :=\sum_{j \in \mathbb{Z}} \beta_{i}\left(\Phi_{j, k, k+1}^{\varepsilon}[\mu]\right) m_{j, k}^{\varepsilon}[\mu], \\
m_{i, 0}^{\varepsilon}[\mu] & :=\int_{E_{i}} m_{0}(x) \mathrm{d} x .
\end{aligned}
$$


where $E_{i}:=\left[x_{i} \pm \frac{1}{2} \rho\right]$ for all $i \in \mathbb{Z}$.

Remark 3.1: The above scheme has the follows probabilistic interpretation: For any $k=0, \ldots, N$, we have that $\left\{m_{i, k} ; 1 \in \mathbb{Z}\right\}$ corresponds to the law of a inhomogeneous discrete-time Markov chain $\left\{X_{k} ; k=0, \ldots, N\right\}$, taking values in $\mathcal{G}_{\rho}$, with initial distribution

$$
\mathbb{P}\left(X_{0}=x_{i}\right)=\int_{E_{i}} m_{0}(x) \mathrm{d} x
$$

and transition probabilities

$$
\mathbb{P}\left(X_{k+1}=x_{i} \mid X_{k}=x_{j}\right)=\beta_{i}\left(\Phi_{j, k, k+1}^{\varepsilon}[\mu]\right) .
$$

Let us define $m_{\rho, h}^{\varepsilon}[\mu]\left(\cdot, t_{k}\right) \in L^{\infty}(\mathbb{R})$ as

$$
m_{\rho, h}^{\varepsilon}[\mu]\left(x, t_{k}\right):=\frac{1}{\rho} \sum_{i \in \mathbb{Z}} m_{i, k}^{\varepsilon}[\mu] \mathbb{I}_{E_{i}}(x)
$$

and if $t \in\left[t_{k}, t_{k+1}\right]$

$$
\begin{aligned}
m_{\rho, h}^{\varepsilon}[\mu](x, t):= & \left(\frac{t_{k+1}-t}{h}\right) m_{\rho, h}^{\varepsilon}[\mu]\left(x, t_{k}\right) \\
& +\left(\frac{t-t_{k}}{h}\right) m_{\rho, h}^{\varepsilon}[\mu]\left(x, t_{k+1}\right) .
\end{aligned}
$$

It is easy to check that $m_{\rho, h}^{\varepsilon}[\mu] \in C\left([0, T] ; \mathcal{P}_{1}\right)$.

\section{The fully discrete approximation SL of the first order} mean field game problem

Given the above discretizations, it is natural so consider the following discrete version of (III.4)

$$
\text { Find } \mu \in C\left([0, T] ; \mathcal{P}_{1}\right) \text { such that } m_{\rho, h}^{\varepsilon}[\mu]=\mu \text {. }
$$

We have (see [9, Theorem 4.12])

Theorem 3.2: There exists at least one solution of (III.13).

The proof of the above result is based on Brouwer's fixed-point theorem. The main result of [9] is the following convergence property for the solutions of (III.13).

Theorem 3.3: Consider a sequence of positive numbers $\rho_{n}, h_{n}, \varepsilon_{n}$ satisfying that $\rho_{n}=o\left(h_{n}\right), h_{n}=o\left(\varepsilon_{n}\right)$ and $\rho_{n}=O\left(\varepsilon_{n}^{3 / 2}\right)$ as $\varepsilon_{n} \downarrow 0$. Let $\left\{m^{n}\right\}_{n \in \mathbb{N}}$ be a sequence of solutions of (III.13) for the corresponding parameters $\left(\varepsilon_{n}, \rho_{n}, h_{n}\right)$. Then every limit point in $C\left([0, T] ; \mathcal{P}_{1}\right)$ of $m^{n}$ (there exists at least one) solves (III.4). In particular, if (II.1) holds we have that $m_{\rho_{n}, h_{n}}^{\varepsilon_{n}} \rightarrow m$ (the unique solution of (MFG)) in $C\left([0, T] ; \mathcal{P}_{1}\right)$ and in $L^{\infty}(\mathbb{R} \times[0, T])$-weak-*.

Remark 3.2: Even if all the properties of our scheme are valid on a general dimension for the state space, we are able to prove the above convergence result only for the one-dimensional case (see [9]). Thus, the proof of a convergence of this type in general dimensions remains as an open question.

\section{A FULLY DISCRETE SL SCHEME FOR THE SECOND ORDER MFG SYSTEM}

In this section we consider the natural extension of the scheme presented above to the second order MFG system

$$
\begin{aligned}
& -\partial_{t} v-\frac{1}{2} \sigma^{2} \Delta v+\frac{1}{2}|D v|^{2}=F(x, m(t)), \text { in } Q, \\
& \partial_{t} m-\frac{1}{2} \sigma^{2} \Delta v-\operatorname{div}(D v m)=0, \quad \text { in } Q, \\
& v(x, T)=G(x, m(T)) \quad \text { for } x \in \mathbb{R}, \quad m(0)=m_{0} .
\end{aligned}
$$

As before, given $\mu \in C\left([0, T] ; \mathcal{P}_{1}\right)$, we denote by $v[\mu]$ the classical solution of the HJB equation (see [12])

$$
\begin{aligned}
& -\partial_{t} v-\frac{1}{2} \sigma^{2} \Delta v+\frac{1}{2}|D v|^{2}=F(x, \mu(t)), \text { in } Q, \\
& v(x, T)=G(x, \mu(T)) \quad \text { for } x \in \mathbb{R}
\end{aligned}
$$

and by $m[\mu]$ the unique classical solution of the following Kolmogorov or Fokker-Planck equation (see [17])

$$
\begin{aligned}
& \partial_{t} m-\frac{1}{2} \sigma^{2} \Delta m-\operatorname{div}(D v m)=0, \text { in } Q, \\
& m(0)=m_{0} \in \mathcal{P}_{1} .
\end{aligned}
$$

Remark 4.1: The solution $m$ of (IV.3) has the following representation formula (see e.g. [10]): consider the stochastic differential equation (SDE)

$$
\begin{aligned}
\mathrm{d} X(t) & =-D v(X(t), t) \mathrm{d} t+\sigma \mathrm{d} W(t), \quad \forall t \in(0, T), \\
X(0) & =\xi,
\end{aligned}
$$

where the law of $\xi$ is given by $m_{0}$. Then, we have that the law of $X(t)$, denoted by $\mathcal{L}(X(t))$, satisfies

$$
m(t)=\mathcal{L}(X(t)) \quad \forall t \in[0, T] .
$$

We now present the schemes for both equations in (IV.1) separately.

\section{A. The fully-discrete scheme for the HJB equation}

We consider the following scheme for (IV.2)

$$
\left\{\begin{array}{l}
v_{i, k}=\hat{S}_{\rho, h}[\mu]\left(v_{\cdot, k+1}, i, k\right) \quad \forall k=0, \ldots, N-1, \\
v_{i, N}=G\left(x_{i}, \mu\left(t_{N}\right)\right),
\end{array}\right.
$$

where $\hat{S}_{\rho, h}[\mu]: B\left(\mathcal{G}_{\rho}\right) \times \mathbb{Z} \times\{0, \ldots, N-1\} \rightarrow \mathbb{R}$ is defined as

$$
\begin{aligned}
& \hat{S}_{\rho, h}[\mu](f, i, k):=\inf _{\alpha \in \mathbb{R}}\left[\frac{1}{2} I[f]\left(x_{i}-h \alpha+\sqrt{h} \sigma\right)+\right. \\
& \left.\frac{1}{2} I[f]\left(x_{i}-h \alpha-\sqrt{h} \sigma\right)+\frac{1}{2} h|\alpha|^{2}\right]+h F\left(x_{i}, \mu\left(t_{k}\right)\right) .
\end{aligned}
$$

This scheme has been proposed in [6] for a second order possibly degenerate Hamilton-Jacobi-Bellman equation, corresponding to an infinite horizon stochastic optimal control problem. The following properties of $\hat{S}_{\rho, h}[\mu]$ are easy to verify.

Proposition 4.1: The following assertions hold true:

(i) [The scheme is well defined] There exists at least one $\alpha \in \mathbb{R}$ that minimizes the r.h.s. of (IV.5). 
(ii) [Monotonicity] For all $v, w \in B\left(\mathcal{G}_{\rho}\right)$ with $v \leq w$, we have that

$$
\hat{S}_{\rho, h}[\mu](v, i, k) \leq \hat{S}_{\rho, h}[\mu](w, i, k) .
$$

(iii) For every $K \in \mathbb{R}$ and $w \in B\left(\mathcal{G}_{\rho}\right)$ we have

$$
\hat{S}_{\rho, h}[\mu](w+K, i, n)=\hat{S}_{\rho, h}[\mu](w, i, n)+K .
$$

(iv) [Consistency] Let $\left(\rho_{n}, h_{n}\right) \rightarrow 0$ (as $n \uparrow \infty$ ) and consider a sequence of grid points $\left(x_{i_{n}}, t_{k_{n}}\right) \rightarrow(x, t)$ and a sequence $\mu_{n} \in C\left([0, T] ; \mathcal{P}_{1}\right)$ such that $\mu_{n} \rightarrow \mu$. Then, for every $\phi \in$ $C^{2}(\mathbb{R} \times[0, T))$, we have

$$
\begin{aligned}
& \lim _{n \rightarrow \infty} \frac{1}{h_{n}}\left[\phi\left(x_{i_{n}}, t_{k_{n}}\right)-\hat{S}_{\rho_{n}, h_{n}}\left[\mu_{n}\right]\left(\phi_{k_{n+1}}, i_{n}, k_{n}\right)\right] \\
& =-\partial_{t} \phi(x, t)-\frac{\sigma^{2}}{2} \Delta \phi(x, t)+\frac{1}{2}|D \phi(x, t)|^{2}-F(x, \mu(t)) .
\end{aligned}
$$

where $\phi_{k}=\left\{\phi\left(x_{i}, t_{k}\right)\right\}_{i \in \mathbb{Z}}$.

We set $v[\mu]$ for the extension of $v_{i, k}$ to $Q$, as in (III.7).

\section{B. The fully-discrete scheme for the Fokker-Planck equation}

As in the first order case, $v_{\rho, h}^{\varepsilon}[\mu]$ denotes the convolution of $v_{\rho, h}[\mu]$ with a smoothing kernel. Let us define

$$
\begin{aligned}
& \Phi_{i, k, k+1}^{\varepsilon,+}[\mu]:=x_{i}-h D v_{\rho, h}^{\varepsilon}[\mu]\left(x_{i}, t_{k}\right)+\sigma \sqrt{h}, \\
& \Phi_{i, k, k+1}^{\varepsilon,-}[\mu]:=x_{i}-h D v_{\rho, h}^{\varepsilon}[\mu]\left(x_{i}, t_{k}\right)-\sigma \sqrt{h} .
\end{aligned}
$$

We consider the following scheme for (IV.3)

$$
\begin{aligned}
m_{i, k+1}^{\varepsilon}[\mu]:= & \frac{1}{2} \sum_{j \in \mathbb{Z}} \beta_{i}\left(\Phi_{j, k, k+1}^{\varepsilon,+}[\mu]\right) m_{j, k}^{\varepsilon}[\mu] \\
& +\frac{1}{2} \sum_{j \in \mathbb{Z}} \beta_{i}\left(\Phi_{j, k, k+1}^{\varepsilon,-}[\mu]\right) m_{j, k}^{\varepsilon}[\mu], \\
m_{i, 0}^{\varepsilon}[\mu]:= & \int_{E_{i}} m_{0}(x) \mathrm{d} x,
\end{aligned}
$$

and we define the extension of the scheme to an element in $L^{\infty}(\mathbb{R} \times[0, T])$ as in (III.11) and (III.12).

Remark 4.2: As in remark 3.1, the scheme (IV.6) has the follows probabilistic interpretation: For any $k=0, \ldots, N$, we have that $\left\{m_{i, k} ; 1 \in \mathbb{Z}\right\}$ corresponds to the law of a inhomogeneous discrete-time Markov chain $\left\{X_{k} ; k=\right.$ $0, \ldots, N\}$, taking values in $\mathcal{G}_{\rho}$, with initial distribution

$$
\mathbb{P}\left(X_{0}=x_{i}\right)=\int_{E_{i}} m_{0}(x) \mathrm{d} x
$$

and transition probabilities

$$
\begin{aligned}
\mathbb{P}\left(X_{k+1}=x_{i} \mid X_{k}=x_{j}\right)= & \frac{1}{2} \beta_{i}\left(\Phi_{j, k, k+1}^{\varepsilon,+}[\mu]\right) \\
& +\frac{1}{2} \beta_{i}\left(\Phi_{j, k, k+1}^{\varepsilon,-}[\mu]\right) .
\end{aligned}
$$

\section{The fully discrete SL approximation of the second order} mean field game problem

Let us consider the following fully SL discretization of (IV.1)

Find $\mu \in C\left([0, T] ; \mathcal{P}_{1}\right)$ such that $m_{\rho, h}^{\varepsilon}[\mu]=\mu$.

Existence of a solution of (IV.7) as well as a convergence result in $C\left([0, T] ; \mathcal{P}_{1}\right)$ can be established using similar techniques as those explored in [9]. However, since the solutions of (IV.1) are regular, we expect to improve this type of convergence and to obtain similar results to those in [1] with the advantage of being able to chose large time steps.

\section{NumericAl Tests}

We present numerical simulations for the first and second order systems. As we will see, these simulations will show different behavior according to the type of model. Given the discrete positive parameters $(\varepsilon, \rho, h)$, we call $\left\{m_{i, k}^{\varepsilon}\right\}$ for the solutions of (III.13) and (IV.7) and we denote by $\left\{v_{i, k}^{\varepsilon}\right\}$ the associated discrete value functions. In order to compute $m_{i, k}^{\varepsilon}$ and $v_{i, k}^{\varepsilon}$, we use a fixed-point iteration method. Let us emphasize that the convergence of such a method has not been proved yet and remains as an important challenge. We consider as initial guess the element in $m^{\varepsilon, 0}$ given by

$$
m_{i, k}^{\varepsilon, 0}=m_{i, 0}^{\varepsilon}=\int_{E_{i}} m_{0}(x) \mathrm{d} x, \quad i \in \mathbb{Z}, k=0, \ldots, N .
$$

Next, for $p=0,1,2, \ldots$, given $m^{\varepsilon, p}$ we calculate $v^{\varepsilon, p+1}$ with the backward schemes (III.5) and (IV.4), taking as $\mu$ the extension of $m^{\varepsilon, p}$ to $C\left([0, T] ; \mathcal{P}_{1}\right)$ defined in (III.11) and (III.12). The element $m^{\varepsilon, p+1}$ is then computed with the forward schemes (III.10) and (IV.6), using a regularizing kernel $\rho_{\varepsilon}$. In the numerical simulations we approximate (III.8) with a discrete convolution and we use a central difference scheme for the gradient. The iteration process is stopped once the quantities

$E\left(v^{\varepsilon, p}\right):=\left\|v^{\varepsilon, p+1}-v^{\varepsilon, p}\right\|_{\infty}, E\left(m^{\varepsilon, p}\right):=\left\|m^{\varepsilon, p+1}-m^{\varepsilon, p}\right\|_{\infty}$,

are below a given threshold $\tau=10^{-3}$.

In the next two subsections, we will solve the first order scheme (III.13) and the second order scheme (IV.7) with a viscosity coefficient $\sigma=0.05$. We consider a space numerical domain given by $\Omega=[0,1]$ and a final time $T=2$. We will take $G=0$ and $F$ of the form (see Remark 2.1)

$$
F(x, m, t)=f(x, t)+V(x, m),
$$

where

$$
\begin{aligned}
V(x, m) & =-10 \int_{\Omega}(y-x)^{2} \mathrm{~d} m(y) \text { and } \\
f(x, t) & =5(x-(1-\sin (2 \pi t)) / 2)^{2} .
\end{aligned}
$$

Interpreting $F$ as the running cost of an average player, the term $f(x, t)=5(x-(1-\sin (2 \pi t)) / 2)^{2}$ constrains the agents to stay close to the point $(1-\sin (2 \pi t)) / 2$ at each time $t$. The term $V(x, m)$ avoid high concentration of the density distribution.

We choose as initial mass distribution:

$$
m_{0}(x)=\frac{\nu(x)}{\int_{\Omega} \nu(x) d x} \quad \text { with } \nu(x)=e^{-(x-0.5)^{2} /(0.1)^{2}}
$$

and as regularizing kernel

$$
\rho_{\varepsilon}(x)=\frac{1}{\sqrt{2 \pi}} e^{-(x-0.5)^{2} / 2 \varepsilon^{2}},
$$

with $\varepsilon=0.3$. We take as space discretization step $\rho=1.6$. $10^{-2}$ and as time step $h=0.02$. 


\section{A. Test 1: first order MFG model $(\sigma=0)$}

At the initial time the density is concentrated around $x=0.5$; due to the term $f(x, t)$ in the running cost the density tends to concentrate around the curve $x(t)=(1-\sin (2 \pi t)) / 2$. At the same time the second term in $V$ avoid $m$ to form high concentration of players, i.e. the players do not want to be surrounded by a large number of players.

Fig. 1 shows the density evolution in the domain $\Omega \times[0, T]$. In Fig. 2, we show the errors $E\left(m^{\varepsilon, p}\right)$ and $E\left(v^{\varepsilon, p}\right)$ of the fixed-point algorithm for the density and the value function.
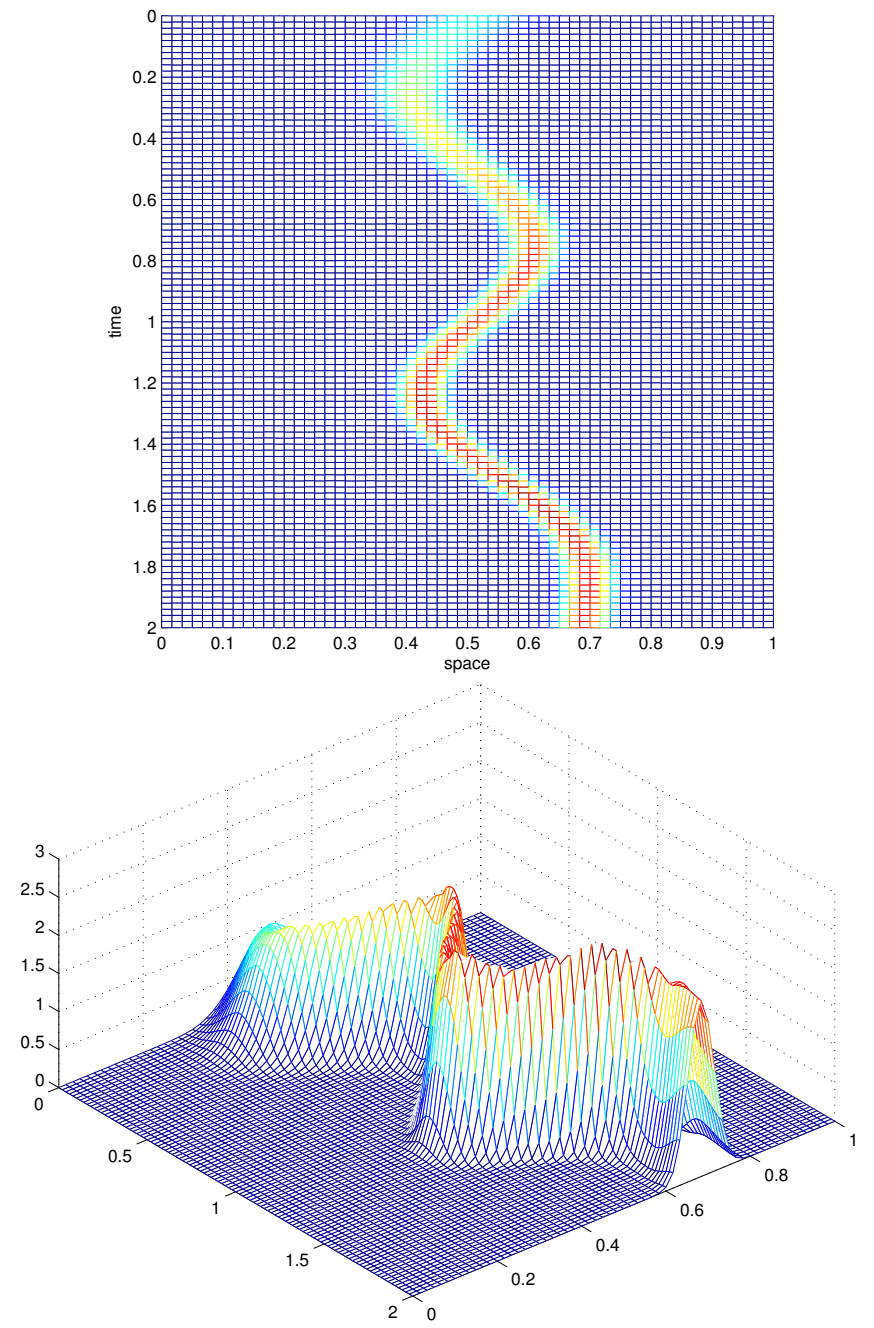

Fig. 1. Density distribution $m_{i, k}^{\varepsilon}$

\section{B. Test 2: second order MFG model $(\sigma=0.05)$}

In this case, the presence of a diffusive term influences the evolution of the density. Again the density tends to concentrate around the curve $x(t)=(1-\sin (2 \pi t)) / 2$. On the other hand, the viscosity term make the density spread around this curve, as it is shown in Fig. 3, where the density evolution is displayed.

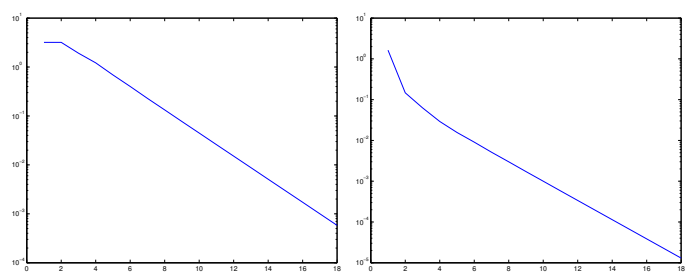

Fig. 2. Errors: $E\left(m^{\varepsilon, p}\right)$ (left), $E\left(v^{\varepsilon, p}\right)$ (right), $p=0 \ldots, 18$
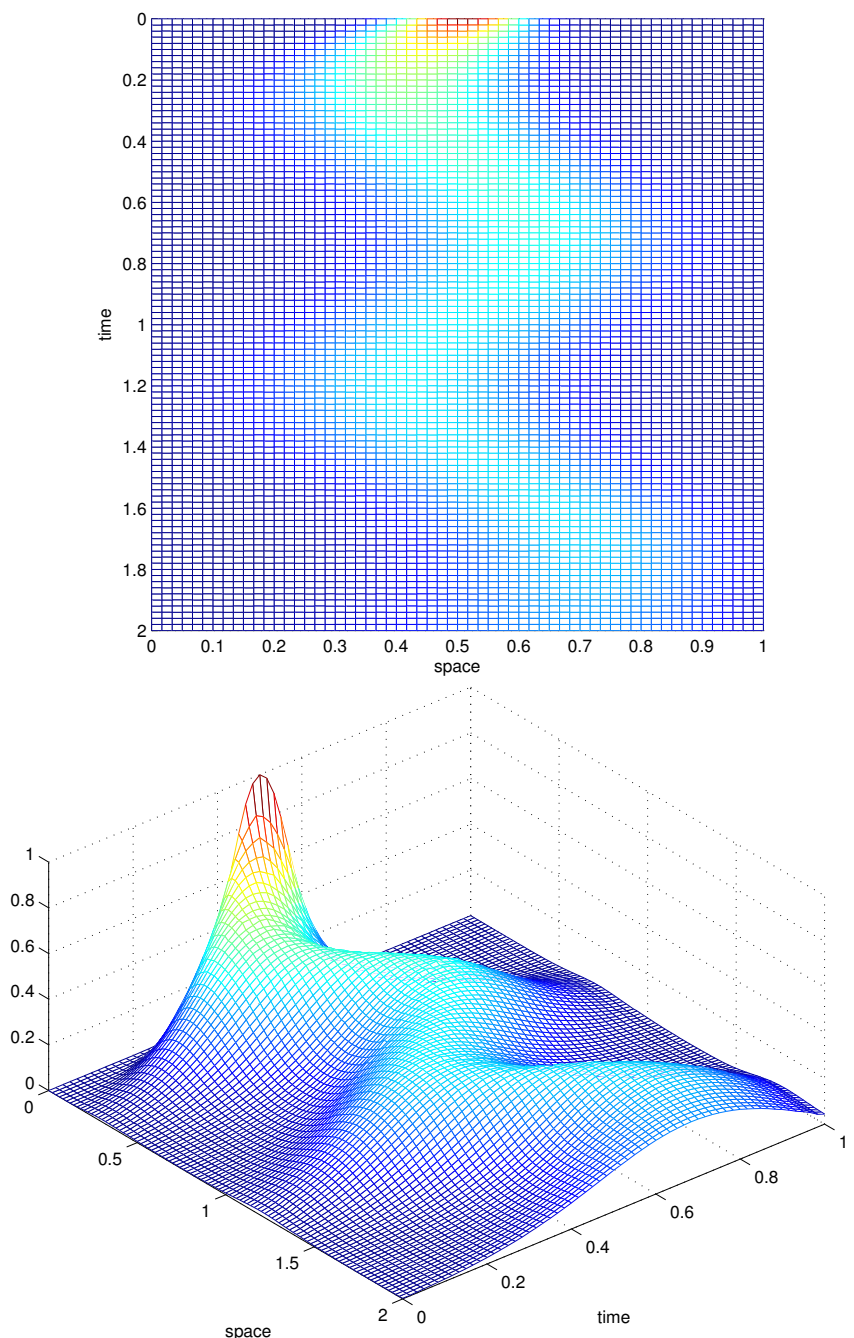

Fig. 3. Density distribution $m_{i, k}^{\varepsilon}$

In Fig. 4 we show the errors $E\left(m^{\varepsilon, p}\right)$ and $E\left(v^{\varepsilon, p}\right)$ of the fixed-point algorithm for the density and the value function.

\section{TOWARDS A GENERALIZATION}

Our scheme can be readily generalized to a more general MFG system of the form

$$
\begin{aligned}
& -\partial_{t} v-\sum_{i, j} a_{i j}(x, t) \partial_{i j} v+H(D v)=F(x, m(t)), \quad \text { in } Q, \\
& \partial_{t} m-\sum_{i, j} \partial_{i j}\left(a_{i j}(x, t) v\right)-\operatorname{div}(D H(D v) m)=0, \quad \text { in } Q, \\
& v(x, T)=G(x, m(T)) \quad \text { for } x \in \mathbb{R}^{d}, \quad m(0)=m_{0},
\end{aligned}
$$


where

$$
a(x, t)=\sigma(x, t) \sigma^{\top}(x, t) .
$$

The existence of a solution of (VI.1) has not been established yet. A possible method to establish such an existence result could be a semi-discrete in time approximation, in the line of [7], and then to pass to the limit when the discretization parameter tends to zero. Even at this early stage, we can always write down the corresponding fully discrete SL schemes for both equations and to couple them in order to figure out the SL scheme for (VI.1). The theoretical and numerical analysis of (VI.1) is a subject of future research of the authors.

\section{CONCLUSIONS}

We have presented a fully discrete SL scheme to approximate both: first and second order MFGs systems. The scheme results to be very flexible, in fact it can be easily generalized to more complicated systems like (VI.1). The main advantage of such scheme is to be explicit and to allow large time steps. In [9], it is shown that for the first order case the following relation between the space and time step is required:

$$
\rho=o(h) .
$$

This is a standard assumption for SL schemes (see the monograph [11] for an extensive description). We have kept this relationship also to approximate the second order problem. Let us remark that the more restrictive parabolic CFL condition ( $h=O\left(\rho^{2}\right)$ ) is avoided. The advantage is that we need much less time iterations to approximate the problem with respect to standard explicit finite difference scheme, for which the parabolic CFL condition is required to make such approximations stable. A rigorous convergence analysis for the SL approximation of the second order problem is the subject of a forthcoming paper.
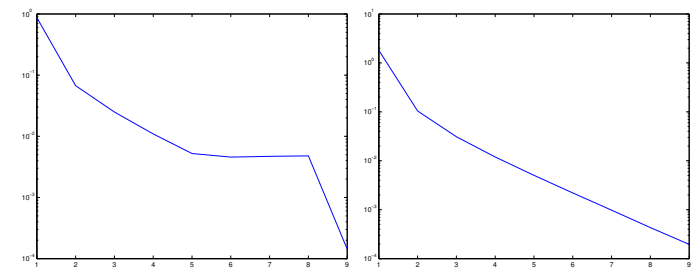

Fig. 4. Errors: $E\left(m^{\varepsilon, p}\right)$ (left), $E\left(v^{\varepsilon, p}\right)$ (right), $p=0 \ldots, 9$

\section{REFERENCES}

[1] Y. Achdou, F. Camilli, and I. Capuzzo Dolcetta. Mean field games: convergence of a finite difference method. Preprint, 2012.

[2] Y. Achdou, F. Camilli, and I. Capuzzo Dolcetta. Mean field games: Numerical methods for the planning problem. SIAM J. of Control \& Optimization, 50:79-109, 2012.

[3] Y. Achdou and I. Capuzzo Dolcetta. Mean field games: Numerical methods. SIAM Journal of Numerical Analysis, 48-3:1136-1162, 2010.

[4] L. Ambrosio, N. Gigli, and G. Savaré. Gradient flows in metric spaces and in the space of probability measures. Second edition. Lecture notes in Mathematics ETH Zürich. Birkhäuser Verlag, Bassel, 2008.

[5] M. Bardi and I. Capuzzo Dolcetta. Optimal control and viscosity solutions of Hamilton-Jacobi-Bellman equations. Birkauser, 1996.
[6] F. Camilli and M. Falcone. An approximation scheme for the optimal control of diffusion processes. RAIRO Modél. Math. Anal. Numér. 29(1):97-122, 1995.

[7] F. Camilli and F. J. Silva. A semi-discrete in time approximation for a first order-finite mean field game problem. Network and Heterogeneous Media, 7-2:263-277, 2012.

[8] P. Cardaliaguet. Notes on Mean Field Games: from P.-L. Lions' lectures at Collège de France. Lecture Notes given at Tor Vergata, 2010

[9] E. Carlini and F.J.Silva. A fully-discrete semi-lagrangian scheme for a first order mean field game problem. Preprint, arXiv:1212.4757, 2012

[10] M. Cessenat, G. Ledanois, P.L. Lions, E. Pardoux, and R. Sentis. Méthodes Probabilistes pour les Equations de la Physique. Edited by R. Dautray. Eyrolles, 1989.

[11] M. Falcone and R. Ferretti. Semi-Lagrangian Approximation Schemes for Linear and Hamilton-Jacobi Equations. MOS-SIAM Series on Optimization, to appear.

[12] W.H. Fleming and H.M. Soner. Controlled Markov processes and viscosity solutions. Springer, New York, 1993.

[13] O. Guéant. Mean field games equations with quadratic hamiltonian: a specific approach. Mathematical Models and Methods in Applied Sciences, 22, 2012.

[14] O. Guéant. New numerical methods for mean field games with quadratic costs. Networks and Heterogenous Media, 7:315 - 336, 2012.

[15] O. Guéant, J.-M. Lasry, and P.-L Lions. Mean field games and applications. In Paris-Princeton Lectures on Mathematical Finance 2010, volume 2003 of Lecture Notes in Math., pages 205-266. Springer, Berlin, 2011.

[16] A. Lachapelle, J. Salomon, and G. Turinici. Computation of mean field equilibria in economics. Mathematical Models and Methods in Applied Sciences, 20-4:567-588, 2010

[17] O. A. Ladyzhenskaya, V.A. Solonnikov, and N.N. Ural'ceva. Linear and quasilinear equations of parabolic type. Translation of Mathematical Monographs, Vol. 23. American Mathematical Society, Providence, R.I., 1967.

[18] J.-M. Lasry and P.-L. Lions. Jeux à champ moyen I. Le cas stationnaire. C. R. Math. Acad. Sci. Paris, 343:619-625, 2006.

[19] J.-M. Lasry and P.-L. Lions. Jeux à champ moyen II. Horizon fini et contrôle optimal. C. R. Math. Acad. Sci. Paris, 343:679-684, 2006.

[20] J.-M. Lasry and P.-L. Lions. Mean field games. Jpn. J. Math., 2:229260, 2007.

[21] P.-L. Lions. Cours au Collège de France. www.college-de-france.fr, 2007-2008 